\title{
Coronary Angiographic Findings Among the Left Bundle Branch Block Patients
}

\author{
ABU TAREK IQBAL 1 , MD AYUB ${ }^{1}$, MD SALEHUDDIN ${ }^{2}$, KHURSHED AHMED ${ }^{3}$ \\ ${ }^{1}$ Department of Medicine, Chattagram Maa O Shishu Hospital Medical College, Chittagong, Bangladesh, ${ }^{2}$ Department of Cardiology, \\ Sher-e-Bangla Medical College, Barishal, Bangladesh, ${ }^{3}$ Department of Cardiology, \\ Bangabandhu Sheikh Mujib Medical University, Dhaka \\ Address of Correspondence: Dr. Abu Tarek Iqbal, Associate Professor, Department of Medicine, Chattagram Maa O Shishu \\ Hospital Medical College, Chittagong, Bangladesh, E-mail: dr.tarekiqbal@yahoo.com
}

\begin{abstract}
Introduction: Complete Left bundle branch block(LBBB) increases the risk of cardiac mortality, and prognosis is primarily determined by the underlying coronary artery diseases. The goal of this study was to determine the association of complete left bundle branch block (LBBB), with site, severity \& risk factors of coronary artery disease (CAD) diagnosed by coronary angiogram(CAG).

Methods: A total of 75 symptomatic patients with LBBB were evaluated in a one year period by coronary angiography in a tertiary care hospital of Chittagong, Bangladesh. Standard protocol and procedure were followed during doing both ECG and CAG. All data were compiled and were analyzed by SPSS-20.

Results: Among 75 patients analyzed male were 40(53.3\%) and female was 35(46.67\%). Male to female ratio was 1:1.14.. Among all 22(29.33\%) patients were at age group $<60$ years, $48(64 \%)$ were at age group 40-60 years and 5(6.6\%) were $<40$ years of age. 34(45.3\%) patients were from rural community. Regarding risk factor analysis 19(25.2\%) patients had DM, 16(21.33\%) patients had different kinds of dyslipidemia, $8(10.6 \%)$ patients were obese, 3(4\%) patients had high cholesterol(>400mg/dl), 26(34.66\%) patients were hypertensive, 21(28\%) were smoker, $2(2.66 \%)$ were taking jarda, $8(10.6 \%)$ patients had family history of coronary artery disease and one patients had history of alcoholism. Angiographic study revealed 6(8\%) had single vessel disease, 16(21.33\%) had double vessel disease, 5(6.66\%) had triple vessel disease and 48(64.00\%) patients had normal vessels. Conclusion: LBBB may be assoictaed with normal to variable involvement of coronary artery
\end{abstract}

\section{Introduction:}

Left bundle branch block (LBBB) usually found in patients with underlying heart disease mainly with ischemic heart although. In a study it was found that $12 \%$ of patients with LBBB have no demonstrable disease. ${ }^{1}$ Even among these patients, LBBB is associated with a higher than normal risk of cardiovascular disease and all cause mortality. ${ }^{1}$

The left bundle branch subdivides into two fascicles: the left anterior fascicle and the left

posterior fascicle. Allowing rapid, coordinated, and synchronous physiologic depolarization of the ventricles. When a bundle branch or fascicle becomes injured, this result in extensive reorganization of the activation and recovery patterns of the left ventricle that produces extensive changes in the QRS complex and the ST-T wave. ${ }^{2}$

Among patients with coronary artery disease, the presence of LBBB correlate with more extensive disease, more severe left ventricular dysfunction, and reduced survival rates. A major impact of LBBB lies in obscuring or simulating other electrocardiographic patterns. ${ }^{3}$

\section{Methods:}

It was a prospective study done on 75 symptomatic patients of $\mathrm{LBBB}$ cases during a study period of one years from July 2015 to December 2015 in a tertiary care center with CCU facility in Chittagong, Bangladesh. Potential patients were initially evaluated who were presented with cardiac complaints. After initial evaluation consent was taken for standard ECG testing. Standard protocol of ECG test was followed during the test and those who LBBB and also symptomatic for IHD were taken as study subjects. In these way a total of 75 patients were recruited. All patients were prepared for coronary angiogram and the test was done in a single center with the direct supervision of the researcher himself. All data were collected and analyzed by SPSS 20 .

\section{Results:}

Among 75 patients analyzed male were 40(53.3\%) and female was $35(46.67 \%)$. Male to female ratio was 1:1.14.. Among all 22(29.33\%) patients were at age group $<60$ years, $48(64 \%)$ were at age group $40-60$ years and $5(6.6 \%)$ were $<40$ years of age. $34(45.3 \%)$ patients 
were from rural community. Regarding risk factor analysis $19(25.2 \%)$ patients had DM, 16(21.33\%) patients had different kinds of dyslipidemia, $8(10.6 \%)$ patients were obese, $3(4 \%)$ patients had high cholesterol $(>400 \mathrm{mg} / \mathrm{dl}), 26(34.66 \%)$ patients were hypertensive, 21(28\%) were smoker, 2(2.66\%) were taking jarda, $8(10.6 \%)$ patients had family history of coronary artery disease and one patients had history of alcoholism. Angiographic study revealed 6(8\%) had single vessel disease, $16(21.33 \%)$ had double vessel disease, 5(6.66\%) had triple vessel disease, and $48(64.00 \%)$ patients had Normal vessel.

Table-I

Sociodemographic profile

\begin{tabular}{llcc}
\hline Variables & Descriptions & Frequency & Percentages \\
\hline Gender & Male & 40 & $53.3 \%$ \\
& Female & 35 & $46.67 \%$ \\
Age group & $<40$ years & 5 & $6.6 \%$ \\
& $40-60$ years & 48 & $64 \%$ \\
& $>60$ years & 22 & $29.33 \%$ \\
Locality & Rural & 34 & $45.3 \%$ \\
& Urban & 41 & $54.7 \%$ \\
\hline
\end{tabular}

TableII

Risk factors

\begin{tabular}{lcc}
\hline Variables & Frequency & Percentages \\
\hline DM & 19 & $25.2 \%$ \\
Dyslipidemia & 16 & $21.33 \%$ \\
Obesity & 8 & $10.6 \%$ \\
High TC $(>400 \mathrm{mg} / \mathrm{dl})$ & 3 & $4.0 \%$ \\
HTN & 26 & $34.66 \%$ \\
Smoking & 21 & $28 \%$ \\
Jorda intake & 2 & $2.66 \%$ \\
Family history of IHD & 8 & $10.6 \%$ \\
Alcohol & 1 & $1.3 \%$ \\
\hline \multicolumn{2}{c}{}
\end{tabular}

Table-III

Angiographic findings of critical lesion

\begin{tabular}{lcc}
\hline Variables & Frequency & Percentages \\
\hline Single vessel disease & 6 & $8 \%$ \\
Double vessel disease & 16 & $21.33 \%$ \\
Triple vessel disease & 5 & $6.66 \%$ \\
Normal & 48 & $64.00 \%$ \\
\hline & 75 & $100 \%$ \\
\hline
\end{tabular}

Multiple response table

\section{Discussion}

In this study patients underwent invasive angiography after finding positive history with ECG findings of LBBB in a tertiary care hospital of Chittagong, Bangladesh during the period from one year study period from January 2016 through December 2016. Among 75 patients analyzed male were $40(53.3 \%)$ and female was $35(46.67 \%)$. Male to female ratio was 1:1.14.. Among all $22(29.33 \%)$ patients were at age group $<60$ years, $48(64 \%)$ were at age group $40-60$ years and $5(6.6 \%)$ were $<40$ years of age. $34(45.3 \%)$ patients were from rural community. These sociodemographic profile are consistent with a Bangladeshi study done by Islam and Majumder. ${ }^{4}$ A higher prevalence of ischemic heart disease in male than female has been reported in a study from England. ${ }^{5}$ Thus the present results are in agreement that male population is more prone to IHD which may be linked to genetic/ hormonal difference.

Regarding risk factor analysis $19(25.2 \%)$ patients had DM, 16(21.33\%) patients had different kinds of dyslipidemia, $8(10.6 \%)$ patients were obese, $3(4 \%)$ patients had high cholesterol( $>400 \mathrm{mg} / \mathrm{dl}), 26(34.66 \%)$ patients were hypertensive, 21(28\%) were smoker, $2(2.66 \%)$ were taking jarda, $8(10.6 \%)$ patients had family history of coronary artery disease and one patients had history of alcoholism. The results of present study with reference to risk factors were similar to those published earlier that diabetes and hypertension are two common risk factors of IHD. Dyslipidemia were also found more. $\mathrm{H} / \mathrm{O}$ smoking, life style and family history of IHD were found as common risk factors. All of the above findings are consistent with the earlier study. ${ }^{6}$

Angiographic study revealed 6(8\%) had single vessel disease, $16(21.33 \%)$ had double vessel disease, $5(6.66 \%)$ had triple vessel disease .

In the present study, we found that and 48(64.00\%) patients had normal coronary angiography. Similarly, Nguyen et al found that $60 \%$ of evaluated patient were presenting with normal angiography. ${ }^{7}$ However, in contrast to our finding, most researchers have shown higher percentage of CAD in patients with $\mathrm{LBBB} .{ }^{8} \mathrm{An}$ explanation for the low prevalence of CAD in $\mathrm{LBBB}$ patients observed in our study was the lack of non invasive diagnostic test such as SPECT study, pharmacological or exercise-induced stress echocardiography of CAD in patients attending our center.

So, it can be concluded that, LBBB be may be associated with various angiographic abnormality in our setting and should be dealt carefully. 


\section{References:}

1. Miller WL, Ballman KV, Hodge DO, Rodeheffer RJ, Hammill SC. Risk factor implication of incidentally discovered uncomplicated BBB. Mayo clinic proc 2005; 80(12): 1585-90

2. Francia P. Left bundle-branch block - Pathophysiology, prognosis and clinical management. Clinical Cardiology. 2007;30:110.

3. Blanc JJ, Fatemi M, Bertault V, Baraket F, Etienne Y. Evaluation of left bundle branch block as a reversible cause of nonischaemic dilated cardiomyopathy with severe heart failure. A new concept of left ventricular dyssynchrony-induced cardiomyopathy. Europace 2005; 7(6): 604-10.

4. Islam IAKM, Majumder A.A.S. Coronary artery disease in Bangladesh: A review. Indian Heart J. 201365(4): pp.424-435
5. Ayub M, Waseem T et al. Risk stratification of patients presenting with first acute myocardial infarction with serum cardiac troponin T. Pak J Card 1999; 10:54-56

6. Esteghamati A et al. Cardiovascular Prevalence of diabetes and other cardiovascular risk factors in an Iranian population with acute coronary syndrome. Diabetol 2006;17:15

7. Nguyen K, Cigarroa JE, Lange RA, Hillis LD, Keeley EC. Presence and extent of angiographic coronary narrowing in patients with left bundle branch block. Am J Cardiol 2004; 93(11):1426-7.

8. Abrol R, Trost JC, Nguyen K, Cigarroa JE, Murphy SA, McGuire $\mathrm{DK}$, et al. Predictors of coronary artery disease in patients with left bundle branch block undergoing coronary angiography. Am J Cardiol 2006;98(10):1307-10. 